Electron Diffraction Experiments using Laser Plasma Electrons

E. E. Fill, S. Trushin, R. Tommasini, R. Bruch

September 19, 2005

Superstrong Fields In Plasmas

Villa Monastero, Varenna, Italy

September 19, 2005 through September 24, 2005 
This document was prepared as an account of work sponsored by an agency of the United States Government. Neither the United States Government nor the University of California nor any of their employees, makes any warranty, express or implied, or assumes any legal liability or responsibility for the accuracy, completeness, or usefulness of any information, apparatus, product, or process disclosed, or represents that its use would not infringe privately owned rights. Reference herein to any specific commercial product, process, or service by trade name, trademark, manufacturer, or otherwise, does not necessarily constitute or imply its endorsement, recommendation, or favoring by the United States Government or the University of California. The views and opinions of authors expressed herein do not necessarily state or reflect those of the United States Government or the University of California, and shall not be used for advertising or product endorsement purposes. 


\title{
Electron Diffraction Experiments using Laser Plasma Electrons
}

\author{
Ernst E. Fill*, Sergei Trushin*, Riccardo Tommasini ${ }^{\dagger}$, \\ and Reinhard Bruch \\ *Max-Planck-Institut für Quantenoptik, D-85748 Garching, Germany \\ ${ }^{\dagger}$ Lawrence Livermore National Laboratory, Livermore, CA 94550, USA \\ "University of Nevada Reno, RenoNV 89557, USA
}

\begin{abstract}
We demonstrate that electrons emitted from a laser plasma can be used to generate diffraction patterns in reflection and transmission. The electrons are emitted in the direction of laser polarization with energies up to $100 \mathrm{keV}$. The broad electron energy spectrum makes possible the generation of a "streaked" diffraction pattern which allows recording fast processes in a single run.
\end{abstract}

\section{INTRODUCTION}

The techniques oltrafast electron- and X-ray diffraction have become powerful tools in the investigation of transient processes in solids and gases [1-4]. Compared with X-rays electrons have the advantage of a much larger scattering cross-section, a shorter wavelength and an applicability to few-electron systems. On the other hand, samples for electron diffraction are more difficult to prepare and to handle, one has to work under high-vacuum conditions and Coulombic repulsion leads to pulse broadening.

In standard time-resolved electron diffraction experiments the electrons are generated by means of a photocatode and then accelerated by an external potential. In this way ultrashort electron pulses with a duration $<1$ ps are generated which can be applied in pump-probe experiments.

In this work we use a different approach, in which the electrons emitted from a laser plasma are utilized. Electron energies obtained using a table top laser at intensities of a few $10^{16} \mathrm{~W} / \mathrm{cm}^{2}$ are well suited for electron diffraction experiments. However, the broad energy range of the generated electrons requires a different technique to be used: instead of carrying out pump probe experiments on deals with an electron streak camera which has the advantage that a single run displays the whole temporal evolution of the effect to be investigated. 
AIP Conference Proceedings.

Third International Conference on

SUPERSTRONG FIELDS IN PLASMAS

Villa Monastero, Varenna (LC), Italy

September 19-24, 2005

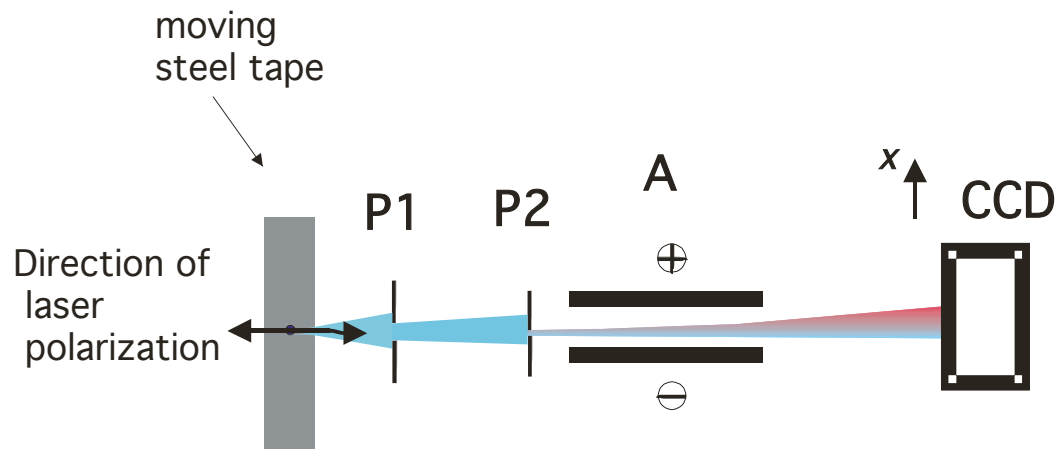

FIGURE 1. Experimental setup for generating collimated electron pulses from a laser plasma. The laser propagation direction is perpendicular to the image plane. P1 and P2 are pinholes $1 \mathrm{~mm}$ and $150 \mu \mathrm{m}$ in diameter. The beam is dispersed by the electrostatic analyzer A and detected by a backside-illuminated X-ray CCD camera.

\section{GENERATING MULTI-KEV ELECTRON PULSES}

The experimental setup has been described previously $[5,6]$. In short, a commercial titanium-sapphire laser emitting $1.6 \mathrm{~mJ}$ in $45 \mathrm{fs}$ at a $1 \mathrm{kHz}$ repetition rate is focused with a $12 \mathrm{~cm}$ focal length lens on a steel tape (Fig. 1). The tape is slowly moving in vertical direction and the electrons are emitted horizontally, along the direction of polarization. To collimate the elctrons and reduce the amount of scattered laser light, two pinholes, $1 \mathrm{~mm}$ and $150 \mu \mathrm{m}$ in diameter are placed in the beam path. In some experiments the second pinhole is opened to a larger diameter. The beam is then dispersed in an electrostatic analyzer consisting of two brass plates separated by $1 \mathrm{~cm}$. As a detector a backside-illuminated soft$\mathrm{X}$-ray CCD is used.

The generated electron spectrum is determined by analyzing the electron streak generated on the CCD (Fig. 2) taking the deflection by the analyzer and the sensitivity of the CCD chip properly into account. As shown in Fig. 3 electrons of up to $100 \mathrm{keV}$ are emitted in the direction of polarization. A plateau in the electron energy distribution is seen at around $40 \mathrm{keV}$ with a flux of $1.5 \times 10^{7}$ electrons per $\mathrm{keV}$ and steradian. Perpendicularly to the laser polarization fewer and colder electrons are emitted.

It is straightforward to generate quasi-monoenergetic electrons by placing a thin pinhole or a slit behind the analyzer. The electron energy can be selected by an appropriate voltage on the analyzer plates. The energy spread can be made arbitrarily small by choosing a narrow slit. In this way, using a $50 \mu \mathrm{m}$ slit electrons with an energy spread of $1 \%$ have been generated at $35 \mathrm{keV}$ [6] 
AIP Conference Proceedings.

Third International Conference on

SUPERSTRONG FIELDS IN PLASMAS

Villa Monastero, Varenna (LC), Italy

September 19-24, 2005

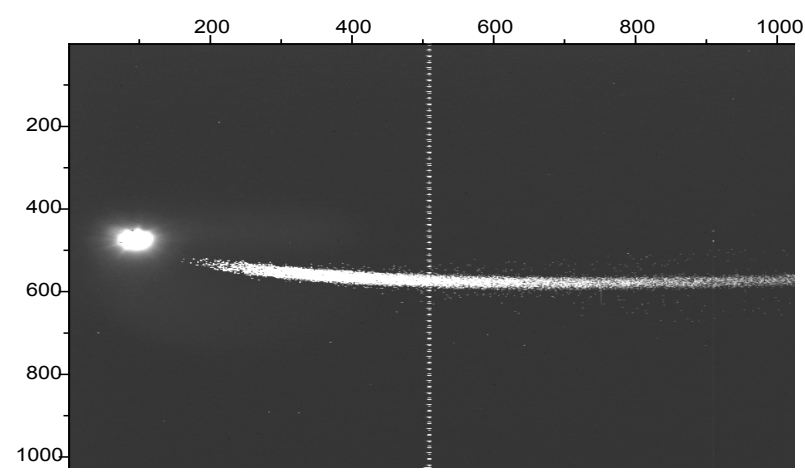

FIGURE 2. CCD image of dispersed electron beam. The bright spot on the left is due to visible light and X-rays. The electrostatic analyzer disperses the electron beam to the right. The slight curvature of the streak is caused by the earth magnetic field. The numbers on the axes display CCD pixel numbers.

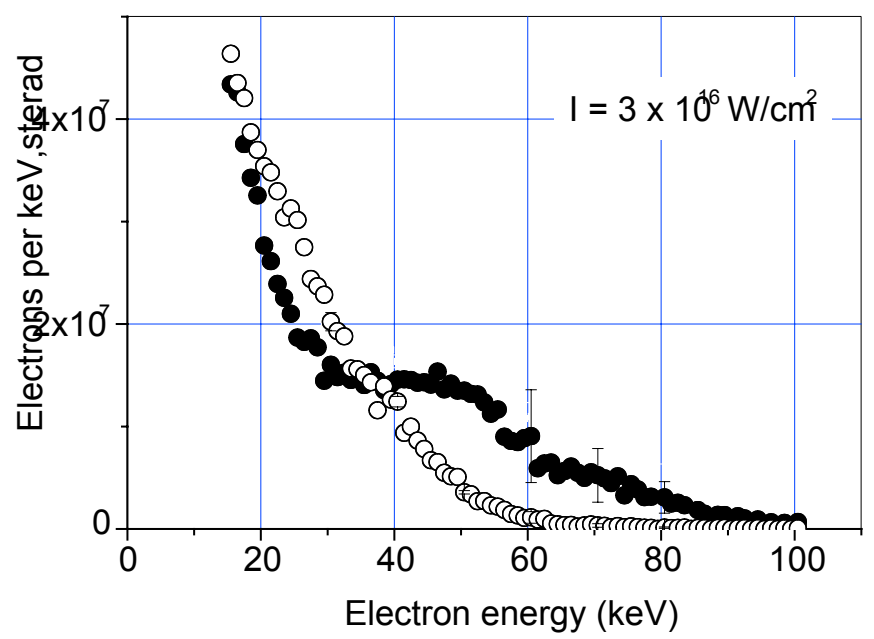

FIGURE 3. Energy distribution of laser-generated electrons at $3 \times 10^{16} \mathrm{~W} / \mathrm{cm}^{2}$. Closed circles display data for laser polarization in the direction of observation. Open circles relate to perpendicular polarization.

\section{DIFFRACTION EXPERIMENTS}

Diffraction experiments using the plasma electrons were carried out using two techniques, RHEED (reflection high-energy electron diffraction) and THEED (transmission high-energy electron diffraction, see for example [7]). For the RHEED experiments the electron beam was scattered off an aluminium pinhole of $1 \mathrm{~mm}$ diameter. Since this technique is surface-sensitive the diffraction pattern is generated by the thin aluminium-oxide layer on top of the bulk material. The dominating diffraction in crystalline hep $\mathrm{Al}_{2} \mathrm{O}_{3}$ is the strong (002) reflection with a $d$-spacing of $1.13 \mathrm{~nm}$. 
AIP Conference Proceedings.

Third International Conference on

SUPERSTRONG FIELDS IN PLASMAS

Villa Monastero, Varenna (LC), Italy

September 19-24, 2005

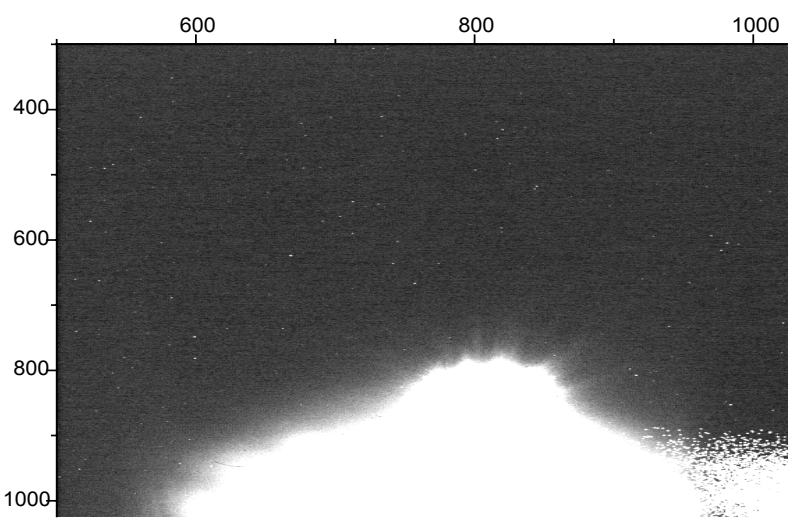

FIGURE 4. CCD image displays RHEED diffraction lobes on the two sides of the main beam. The beam is passed through an electrostatic lens which focuses a certain electron energy. Lobes above and below the beam display diffraction from an aluminium pinhole. The lobes separate farther from the main beam the lower the electron energy, reflecting the longer de Broglie wavelength.

Dispersing the electrons after the diffracting element again leads to an electron streak, which now exhibits a diffraction lobe on each side (Fig. 4). Their distance from the main streak increases with decreasing electron energy. An electrostatic lens was introduced in the beam path after the diffracting element in order to enhance the intensity. The lens is highly dispersive and focuses only electrons with a specific electron energy. Note that the X-axis of the CCD is also a time axis, since electrons of different energies have different velocities. With the parameters of the present experiment the time-window of the image is about a ns
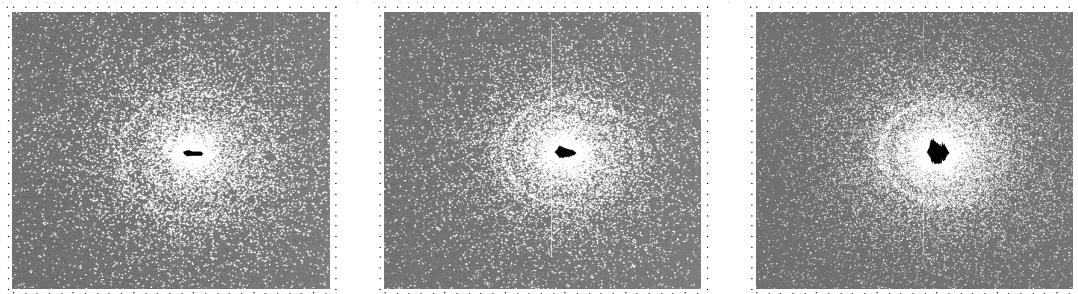

FIGURE 5. Debye-Scherrer rings generated by diffraction of an electron beam by a thin aluminium foil. The central beam energies are from left to right 45, 53 and $67 \mathrm{keV}$ respectively with an energy spread of $20 \%$. The black beam center is overexposed. The asymmetry of the patterns is caused by the energy range of the electrons (see text).

For the THEED experiments a third pinhole, with a diameter of $150 \mu \mathrm{m}$ was introduced after the analyzer, by $5 \mathrm{~mm}$ offset from the axis. A commercial electron diffraction sample was placed behind this pinhole. A powder (Debye- 
AIP Conference Proceedings.

Third International Conference on

SUPERSTRONG FIELDS IN PLASMAS

Villa Monastero, Varenna (LC), Italy

September 19-24, 2005

Scherrer) diffraction pattern was generated on the CCD in a few minutes of acquisition. The energy of the electrons traversing the sample can be chosen by an appropriate voltage on the analyzer. Fig. 5 shows a series of diffraction patterns with electron energie ranging from 45 to $67 \mathrm{keV}$. The diameters of the rings contract as the energy is increased, in accord with the decreasing de Broglie wavelength of the electrons. The asymmetry of the diffraction patterns is explained by fact that the electrons have a range of energies: The beam center is slightly elongated with the low-energy part on the right hand side. Therefore the rings coincide on the left part of the pattern and are smeared over a larger area on the right hand side.

\section{FUTURE INVESTIGATIONS}

The generated electron pulse can be applied to investigate fast processes in a single run. Any change in the electron beam parameters is noticeable in the streak along the $\mathrm{x}$-axis of the detector. Note that the velocity dispersion of the "chirped" electron pulse results in a temporal spread as the pulse propagates. For example for a $10 \%$ energy spread at $60 \mathrm{keV}$ the pulse lengthens by $3 \mathrm{ps}$ for each $\mathrm{cm}$ of propagation. This feature can be used to advantage to generate an electron streak camera which exhibits "time-magnification": Any ultrafast process imposed on the beam early in its path is displayed in slow motion after a distance of propagation.

It should be mentioned that the delicate character of the samples excludes accumulation of many shots to investigate irreversible processes in a solid. However interesting reversible processes subsist, such as melting and recristallization, or reversible conformational changes in molecular crystals [8]. In gases irreversible processes can be studied as well, such as ionization, Coulomb explosion and photodissociation.

\section{ACKNOWLEDGEMENTS}

We thank W. Fölsner for help in handling the samples. R. Bruch is grateful to the University of Nevada Reno for granting him sabbatical leave. S. Trushin thanks Deutsche Forschungsgemeinschaft for a research fellowship (project FU 363/1). R. Tommasini's work was performed under the auspices of the U.S. Department of Energy by University of California, Lawrence Livermore National laboratory under Contract W-7405-Eng-48. This work was supported in part by the Commission of the European Communities in the framework of the Euratom/Max-Planck-Institut für Plasmaphysik Association 
AIP Conference Proceedings.

Third International Conference on

SUPERSTRONG FIELDS IN PLASMAS

Villa Monastero, Varenna (LC), Italy

September 19-24, 2005

\section{REFERENCES}

[1] J. R. Helliwell and P. M. Rentzepis, Time-resolved Diffraction (Clarendon Press, Oxford, 1997).

[2] H. Ihee, V. A. Lobastov, U. M. Gomez, B. M. Goodson, R. Srinivasan, C.-Y. Ruan, and A. H. Zewail, Science 291, 458 (2001).

[3] R. Srinivasan, V. A. Lobastov, C.-Y. Ruan, and A. H. Zewail, Helv. Chim. Acta 86, 1763 (2003).

[4] B. J. Siwick, J. R. Dwyer, R. E. Jordan, and R. J. Dwayne Miller, Science 302, 1382 (2003).

[5] E. E. Fill, S. Trushin, R. Bruch, and R. Tommasini, Appl. Phys. B 81, 155 (2005).

[6] R. Tommasini, E. Fill, R. Bruch, and G. Pretzler, Appl. Phys. B 79, 923 (2004).

[7] L. E. Murr, Electron and Ion Microscopy and Microanalysis (Marcel Dekker, Inc., New York, 1982).

[8] R. Tommasini, C. Root, P. Gilch, M. Braun, W. Zinth, and E. Fill, in LaserGenerated and Other Laboratory X-ray and XUV Sources, edited by G. A. Kyrala, J.-C. Gauthier, C. A. MacDonald and A. M. Kounsary (SPIE, San Diego, 2003), Vol. 5196, p. 35. 